\title{
Frequency of a very brief intervention by physiotherapists to increase physical activity levels in adults: a pilot randomised controlled trial
}

\author{
Nicole Freene ${ }^{1 *}$ D, Rachel Davey ${ }^{2}$ and Steven M McPhail ${ }^{3,4}$
}

\begin{abstract}
Background: There is evidence that brief physical activity interventions by health professionals can increase physical activity levels. In addition, there is some evidence that simply measuring physical activity alone can increase physical activity behaviour. However, preliminary work is required to determine the effects of potential measurement frequency. The aim of this pilot study was to examine whether frequency of physical activity measurement, with very brief advice from a physiotherapist, influenced objectively measured physical activity in insufficiently active adults.

Methods: Using concealed allocation and blinded assessments, eligible participants $(n=40)$ were randomised to a lower-measurement-frequency (baseline and 18-weeks) or higher-measurement-frequency group (baseline, 6, 12 and 18-weeks). The primary outcome was daily minutes of moderate-to-vigorous physical activity (accelerometry). Secondary outcomes included functional aerobic capacity (STEP tool), quality-of-life (AQoL-6D), body mass index, waist circumference, waist-to-hip ratio and blood pressure.

Results: Between-group comparisons were not significant in intention-to-treat analyses. However, there was a trend for the higher-measurement-frequency group to complete more daily minutes of moderate-to-vigorous physical activity at 18-weeks (mean difference 19.6 vs -11.9 mins/week, $p=0.084$ ), with a medium effect size (Cohen's $d=0.58$ ). This was significant in per-protocol analysis ( $p=0.049$, Cohen's $d=0.77$ ). Within-group comparisons indicated both groups increased their aerobic fitness ( $p \leq 0.01$ ), but only the higher-measurement-frequency group decreased their waist circumference (mean decrease $2.3 \mathrm{~cm}, 95 \% \mathrm{Cl} 0.3-4.3, p=0.024$ ), diastolic blood pressure (mean decrease $3.4 \mathrm{mmHg}$, $95 \% \mathrm{Cl} 0.03-6.8, p=0.048$ ) and improved their quality-of-life for independent living (mean increase 3.3, 95\% Cl 0.2-6.4, $p=0.031$.

Conclusion: Very brief physical activity interventions by physiotherapists may be an efficient approach to increase physical activity in community-dwelling adults. A larger trial is warranted.

Trial registration: Australian New Zealand Clinical Trials Registry (ANZCTR): ACTRN12616000566437, http://www. ANZCTR.org.au/ACTRN12616000566437.aspx, registered 2 May 2016.
\end{abstract}

Keywords: Behaviour change, Fitness, Health status, Accelerometry, Measurement

\footnotetext{
* Correspondence: nicole.freene@canberra.edu.au

'Physiotherapy, Faculty of Health, University of Canberra, Bruce 2617,

Australia

Full list of author information is available at the end of the article
}

(c) The Author(s). 2019 Open Access This article is distributed under the terms of the Creative Commons Attribution 4.0 International License (http://creativecommons.org/licenses/by/4.0/), which permits unrestricted use, distribution, and reproduction in any medium, provided you give appropriate credit to the original author(s) and the source, provide a link to the Creative Commons license, and indicate if changes were made. The Creative Commons Public Domain Dedication waiver (http://creativecommons.org/publicdomain/zero/1.0/) applies to the data made available in this article, unless otherwise stated. 


\section{Background}

One quarter of adults worldwide report they are not doing enough physical activity to meet the minimum recommended guidelines for health benefits, and in Australia, the country of this study, the situation is less favourable, with approximately half of adults reporting insufficient levels of physical activity $[1,2]$. Internationally there is a focus on the development and refinement of physical activity interventions as insufficient physical activity is a major risk factor for chronic disease and death [3-6]. Physical activity promotion by health professionals is one type of physical activity intervention that is viewed as a key strategy to improve the populations' physical activity levels. This strategy has been outlined in action plans around the world, and there is some evidence that brief physical activity interventions by health professionals are just as effective as more intensive interventions [7-10].

Health professionals, such as physiotherapists, are well placed to promote and assess physical activity. Physiotherapists are effective communicators, establishing rapport, gaining trust, supporting and empowering an individual [11]. Physiotherapists perceive the provision of physical activity advice as part of their role, and there is evidence that physiotherapists can counsel effectively for physical activity behaviour change and also treat any underlying conditions that may impair someone's physical capacity to be physically active [12-14]. Kunstler et al. (2017) found in a systematic review that adults receiving a physiotherapist-led physical activity intervention in private practice, primary care or outpatient settings doubled their odds of increasing their physical activity levels up to 1 year after the intervention. Yet, internationally it appears that physical activity levels are not routinely assessed by physiotherapists and brief physical activity interventions are not routinely delivered in clinical practice, with lack of time reported as the most commonly perceived barrier $[13,15,16]$.

Interestingly, prior research has indicated simply measuring physical activity levels in control groups, as part of randomised controlled studies investigating physical activity interventions, has been found to increase physical activity behaviour [17]. Waters et al. (2012) found that approximately one third of physical activity intervention studies in primary care have reported improvements in physical activity among participants who were in the control group. In fact, some control groups have been found to increase their physical activity levels to a similar level as the intervention group, particularly over the longer term $[18,19]$. Measurement reactivity, the Hawthorne effect, higher intensity of contact compared with a clinical setting and motivated volunteers are possible explanations for these findings [17, 20, 21]. This may indicate with minimal contact and resources, physical activity behaviour change may be achievable in a proportion of the population. Although, of the 29 studies included in the Waters et al. (2012) systematic review, only four measured physical activity objectively (accelerometry), and this was not included as an outcome in this review. Similarly, Lamming et al. (2017) in their systematic review of reviews found that brief interventions (less than $30 \mathrm{~min}$ ) can increase self-reported physical activity in the short-term (4-12-weeks), and they recommended that future research should focus on very brief interventions (less than $5 \mathrm{~min}$ ) [22]. Thus, frequent measurement of physical activity, with very brief advice by health professionals, in routine clinical care may be a very brief intervention that can result in favourable increases in physical activity levels.

With the ever increasing access to objective activity monitoring devices (accelerometers in smartphones, wrist, shoe or arm worn devices), combined with the broad reach of health professionals, even a modest effect of measurement and very brief advice, with accountability to their health professional, may prove to be an efficient and effective approach to increase the populations' physical activity levels in a meaningful way. However, preliminary work is required to determine the effects of potential measurement and very brief advice frequency. The aim of this study was to examine whether frequency of physical activity measurement and very brief advice provided by physiotherapists increased physical activity levels in a sample of insufficiently active Australian adults.

\section{Methods}

A pilot (two parallel arm, 1:1 allocation ratio) randomized control study was conducted between July and November 2016. Participants were recruited to two, or four, health and fitness assessments at an outpatient clinic using magazine, electronic media and poster advertising. All individuals that responded to the advertisements were contacted by email or telephone by the principal researcher to determine their eligibility. Eligible participants were between 18 and 64 years old, insufficiently active (less than $150 \mathrm{~min}$ of self-reported moderate-to-vigorous physical activity (MVPA) per week), had no serious medical conditions that could limit participation in moderate physical activity, no severe functional impairments due to medical and psychiatric conditions, and adequate English and cognitive skills to participate in the study. Only one person per household was eligible and participants were not planning to move from the city within the 18-week study period. Medical clearance screening was undertaken using the Sports Medicine Australia Pre-Exercise Screening System [23]. All participants provided written consent prior to completion of their baseline assessment.

An investigator who was at a remote location and not involved in recruitment or assessments used a computer to generate a random number sequence and concealed 
group allocation using sealed, consecutively numbered opaque envelopes. Following each participant's baseline assessment the next envelope in the sequence was opened to reveal random allocation to one of two groups: lower-measurement-frequency (LMF; 2 physiotherapist-led physical activity measurements at baseline and week 18) or higher-measurement-frequency (HMF; 4 physiotherapist-led physical activity measurements at baseline, week 6,12 and 18).

\section{Very brief physical activity intervention}

At all assessments participants were given an indication of their fitness, that is, predicted maximum oxygen consumption $\left(\mathrm{VO}_{2} \mathrm{max}\right)$ based on the Step Test and Exercise Prescription (STEP) tool [24]. At baseline, participants received two brochures 'Make your Move Sit less - Be active for life!' [25] and 'Healthy Eating for Adults' [26]. The physical activity guidelines were briefly discussed $(2 \mathrm{~min})$ at each assessment but participants did not receive any other advice or support for increasing exercise or physical activity levels. Examples of the brief physical activity advice provided are 'moderate intensity physical activity means you should be able to talk in full sentences but not sing', and ' $30 \mathrm{~min}$ of moderate physical activity can be accumulated in $10 \mathrm{~min}$ bouts'. All participants were encouraged to increase their physical activity levels safely during the 18-week period aiming to progress towards the public health physical activity guidelines, that is, accumulate 150 to $300 \mathrm{~min}$ of moderate intensity physical activity or 75 to $150 \mathrm{~min}$ of vigorous intensity physical activity or a combination of both each week [27]. The total time required for the STEP tool (mean test length $=70 \pm 15 \mathrm{~s}$ ) and provision of brief advice was less than $5 \mathrm{~min}$, classifying it as a very brief intervention [28].

\section{Outcome measures}

The assessor at baseline and 18 weeks for all participants was blinded to group allocation. To maintain blinding, a different assessor was used for the week 6 and week 12 assessments for the higher-measurement-frequency group (which were not analysed). The higher-measurement-frequency assessor was trained in all measurement procedures, risk communication and brief physical activity advice to ensure standardisation, although one assessor may have been more supportive than the other.

A triaxial commercial accelerometer (ActiGraph GT3X or ActiSleep, Fort Walton Beach, FL) was used to objectively assess physical activity. Participants were given the accelerometer during each assessment and asked to wear the monitor on their right hip, while awake, for 7 -consecutive days, retuning the accelerometer via mail in the reply-paid post pak provided. All data was downloaded and screened, excluding data if: $<10 \mathrm{~h}$ per day wear time (non-wear defined as $>60$ consecutive minutes where there is zero activity, allowing for $2 \mathrm{~min}$ of counts between 0 and 100) and less than 4 days of valid data. The raw data collected by the accelerometer, counts, was then used to obtain the time spent in different physical activity intensities. The Freedson Combination energy expenditure algorithm was used to determine the physical activity intensity cut-points [29]. MVPA bout data used a minimum bout length of $10 \mathrm{~min}$, allowing for 2 min of counts less than the MVPA threshold within this time. Estimating time spent in physical activity was calculated by dividing the total time spent (minutes) in each threshold by the number of valid days. Adherence to the physical activity recommendations (sufficient time) was calculated for $1 \mathrm{~s}$ epoch and $10 \mathrm{~min}$ bout data [27]. Calculations were based on estimates of daily minutes in MVPA, weighting vigorous physical activity by 2 for $1 \mathrm{~s}$ epoch data, and multiplying by 7 to estimate a week.

The Active Australia Survey (AAS) has been designed to measure participation in leisure time physical activity and to assess the participant's knowledge of current public health messages about the health benefits of physical activity [30]. It offers a short and reliable set of questions and applies to 1 week preceding the interview, including walking for transport. The AAS has evidence to support its reliability and validity [31, 32].

The STEP tool was used to assess functional aerobic capacity. The STEP tool is an indirect measure of aerobic capacity, requiring a set of $2 \times 20 \mathrm{~cm}$ steps and is valid for use with adults aged $18-85$ years $[24,33]$. Participants step up and down a standardized set of 2 steps, $20 \mathrm{~cm}$ each, 20 times at a self-selected normal pace following a demonstration of the test. Time to complete the test (secs) and post-test heart rate (bpm) are recorded, calculating predicted $\mathrm{VO}_{2} \max (\mathrm{ml} / \mathrm{kg} / \mathrm{min})$ using these measures and age (yrs), sex, and body weight $(\mathrm{kg}) ; \quad \mathrm{VO}_{2} \max =3.9+(1511 / \text { time })^{*}\left((\text { weight } / \mathrm{HR})^{*} 0.124\right)$ -(age*0.032)-(sex*0.633) [24]. Normative values for classifying $\mathrm{VO}_{2} \max$ (poor-superior) were used to give the participant an indication of their aerobic fitness [24].

The Assessment of Quality of Life (AQoL)-6D is a health-related quality of life questionnaire that was self-administered. The AQoL-6D has 20 items with 4-6 levels, takes 2-3 min for participants to complete, and has six dimensions - Independent Living, Mental Health, Coping, Relationships, Pain, Senses, as well as an overall multi-attribute utility score that can be derived from AQoL responses. The AQoL-6D was scored using the recommended Algorithm for Adults, where a score of 100 reflects best health [34]. The AQoL has evidence supporting its reliability and validity in community settings $[35,36]$. 
Height $(\mathrm{m})$, weight $(\mathrm{kg})$ and body mass index (BMI, kg/ $\mathrm{m}^{2}$ ) were recorded using a calibrated set of scales and a stadiometer. Waist circumference and hip circumference were measured in centimetres using a tape measure, and waist-to-hip ratio was calculated. Resting blood pressure was measured using a mercury sphygmomanometer on the right arm of seated subjects. Sociodemographic information was also collected with questions regarding participant's education level, relationship status, current employment status and the presence of any chronic diseases.

\section{Sample size}

An important purpose of this study was to provide an estimate of the potential effect size of measuring physical activity at two different frequencies over an 18-week period, and there was no prior appropriate data assessing the change in physical activity levels using accelerometey on which to base a sample size estimate for between group comparisons [37]. Therefore, the study sought to recruit 40 participants for this purpose. Data from this study will be used to inform planning for future studies.

\section{Data analysis}

Analyses were carried out following intention-to-treat principles. As a conservative approach for missing data at 18-weeks, no change from baseline was assumed for the intention-to-treat analyses. In addition, a less conservative approach (per-protocol analysis) was completed using only participants who had completed scheduled assessments. Descriptive analyses were also completed. Normality of the data was assessed using the Kolmogorov-Smirnov test. For between group analyses, unpaired t-tests were used for data that was normally distributed, the Mann-Whitney U test was used for data that was not normally distributed, and Chi-squared analysis was used for categorical data. For within group analyses, changes in variables with normal distributions were analysed with paired $t$-tests, if variables were not normally distributed the Wilcoxon Signed Ranks tests were used, and the McNemar test was used for changes in proportions. All analyses were conducted using SPSS version 23. Significance level was set at $p<0.05$.

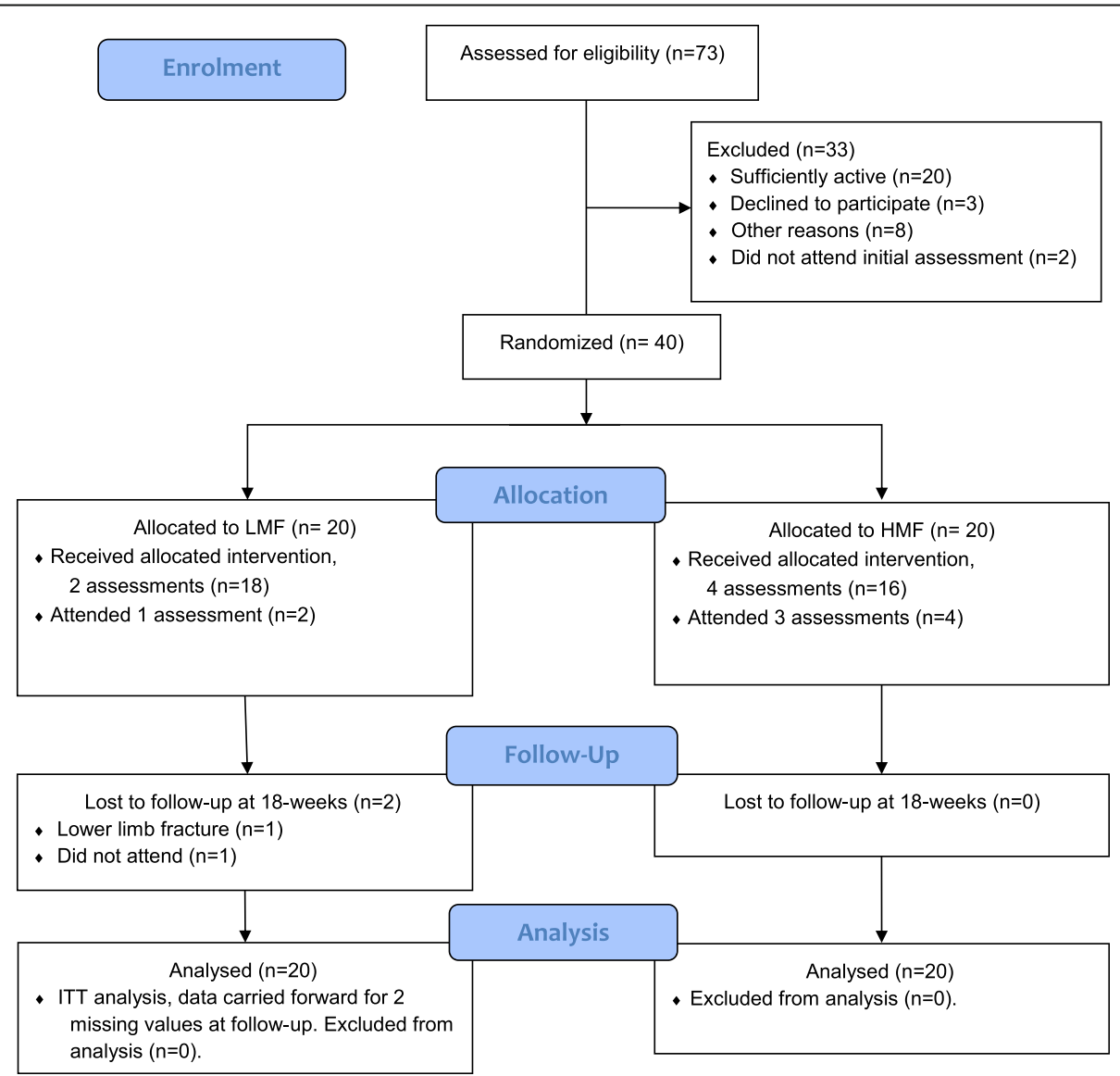

Fig. 1 Flow of participants. HMF, higher-measurement-frequency, 4 physical activity measurements and very brief advice (intervention); LMF, lower-measurement-frequency, 2 physical activity measurements and very brief advice (control); ITT, intention-to-treat 


\section{Results}

Forty participants were recruited (Fig. 1), with a mean age of 44 years, and age range from 20 to 63 years old. Participant characteristics are reported in Table 1. At baseline, there were no differences between groups in objectively measured physical activity. Physical activity measurements are reported in Table 2. Measures of disease risk, fitness and health-related quality of life are reported in Table 3.

At 18 -weeks $95 \%$ (38/40) of participants completed assessments (Fig. 1). Thirty-six participants provided valid accelerometer results at baseline, and 30 participants had valid accelerometer results at 18 -weeks. There was no significant difference between groups for accelerometer wear time, with participants wearing the accelerometers for approximately $14.5 \mathrm{~h}$ per day, for a mean of 6.9 days.

The intention-to-treat analysis indicated there was no significant difference between or within groups in physical activity levels, both self-reported and measured with the accelerometer, at 18-weeks (Table 2). However, there was a trend for participants in the highermeasurement-frequency group to complete more accelerometer daily minutes of MVPA than the lower-measurement-frequency group at 18-weeks, with a medium effect size (Table 2, $p=0.084$, Cohen's $d=0.58$ ). This corresponded to a mean increase of 19.6 MVPA minutes/week in the highermeasurement-frequency group versus an 11.9 MVPA minutes/week decrease in the lower-measurementfrequency group. This finding was significant in the per-protocol analysis comparison between groups at 18-weeks, with the higher-measurement-frequency completing significantly more accelerometer daily minutes of MVPA ( $1 \mathrm{~s})$ than the lower-measurementfrequency group $(p=0.049$, Cohen's $\mathrm{d}=0.77)$.

Both groups significantly increased their aerobic fitness $(p \leq 0.01)$. Although, only participants who were in the higher-measurement-frequency group significantly

Table 1 Participant characteristics at baseline

\begin{tabular}{lll}
\hline Characteristic & \multicolumn{2}{l}{ Baseline } \\
\cline { 2 - 3 } & HMF & LMF \\
\hline Age in years, mean (SD) & $46(14)$ & $41.5(14.8)$ \\
Gender, n females (\%) & $17(85)$ & $16(80)$ \\
Country born, n Australia (\%) & $16(89)$ & $16(80)$ \\
Employment, n paid work (\%) & $12(71)$ & $12(63)$ \\
Education level, n tertiary (\%) & $15(83)$ & $16(80)$ \\
Relationship status, n partner (\%) & $12(67)$ & $16(80)$ \\
Chronic disease, n no (\%) & $15(83)$ & $20(100)$ \\
Blood pressure medication, n no (\%) & $16(89)$ & $19(95)$ \\
\hline
\end{tabular}

HMF, higher-measurement-frequency, 4 physical activity measurements and very brief advice (intervention); LMF, lower-measurement-frequency, 2 physical activity measurements and very brief advice (control) decreased their waist circumference (mean decrease 2.3 $\mathrm{cm}, 95 \% \mathrm{CI}$ 0.3-4.3, $p=0.024$ ), diastolic blood pressure (mean decrease $3.4 \mathrm{mmHg}$, 95\%CI $0.03-6.8, p=0.048$ ) and improved their quality of life independent living domain (mean improvement 3.3, 95\%CI 0.2-6.4, $p=0.031$ ). There were no adverse events recorded.

\section{Discussion}

This is the first randomised trial to report the effect of frequency of physical activity measurement and very brief advice by physiotherapists on objective physical activity levels. These preliminary findings suggest that frequent physical activity measurements with very brief advice by physiotherapists may result in increased objectively measured physical activity. This may also be associated with improvements in other health outcomes such as decreasing waist circumference and diastolic blood pressure, and improving quality-of-life. Importantly, regardless of physical activity measurement frequency, participants significantly increased their aerobic fitness. Further research is indicated to confirm these findings.

van Sluijs et al. (2006) compared different frequencies of physical activity measurement in a large randomised controlled study in Dutch general practice $(n=635)$. Participants were randomised to 3 or 1 physical activity measurements over 6 months. More participants in the 3 physical activity measurement group met the physical activity guidelines at the end of the intervention period as compared to the 1 physical activity measurement group when considering self-reported physical activity but there was no difference found between groups for a sub-sample of participants using accelerometry. The authors concluded that the increased frequency of physical activity measurements affected participants' physical activity behaviour. However, these findings ought to be interpreted cautiously as there was limited self-reported physical activity data collected at baseline for comparison and no baseline objective measurement of physical activity. The present study has extended the field by reporting for the first time a trend for an increase in both subjective and objective measurement of physical activity in the higher-measurement-frequency group (Table 2).

Consideration must be given to the length of the physical activity intervention if it is to be feasible in a health care setting. Lack of time is consistently reported as a barrier to physical activity promotion by physiotherapists [38]. For physical activity promoting interventions to be translated into clinical practice, they must be brief, whether or not they include measurement [22]. This study has reported on a very brief intervention including measurement of functional aerobic capacity with feedback and physical activity advice, recognising that 
Table 2 Physical activity characteristics at baseline and 18-weeks

\begin{tabular}{|c|c|c|c|c|}
\hline \multirow[t]{2}{*}{ Characteristic } & \multicolumn{2}{|l|}{ Baseline } & \multicolumn{2}{|l|}{ 18-weeks } \\
\hline & $\mathrm{HMF}$ & LMF & HMF & LMF \\
\hline \multicolumn{5}{|l|}{ MVPA minutes/day, mean (SD) } \\
\hline Actigraph $1 \mathrm{~s}$ & $64.7(31)$ & $53.7(20.2)$ & $68.4(33.7)$ & $52.6(18.7)$ \\
\hline Actigraph $10 \mathrm{~min}$ bout & $15.4(19.1)$ & $13(10.3)$ & $19.5(31.2)$ & $10.1(10.8)$ \\
\hline Self-report (AAS) & $49(37.2)$ & $44.5(38.8)$ & $51.4(42.5)$ & $40.1(27.3)$ \\
\hline \multicolumn{5}{|l|}{ Sufficient PA time, $n(\%)$} \\
\hline Actigraph $1 \mathrm{~s}^{\#}$ & $18(100)$ & $18(100)$ & $19(100)$ & $19(100)$ \\
\hline Actigraph $10 \mathrm{~min}$ bout & $3(16.7)$ & $3(16.7)$ & $4(21.1)$ & $3(16.7)$ \\
\hline Self-report (AAS) ${ }^{\#}$ & $15(83.3)^{\mathrm{A}}$ & $15(75)$ & $13(68.4)$ & $14(73.7)$ \\
\hline VM counts/day, mean (SD) & $587,087(223086)$ & 511,455 (131679) & $615,223(278145)$ & $504,438(138266)$ \\
\hline
\end{tabular}

${ }^{\mathrm{A}}$ unpaired comparison between groups, $p \leq 0.01$; " vigorous physical activity multiplied by 2 . HMF higher-measurement- frequency, 4 physical activity measurements and very brief advice (intervention), LMF lower-measurement-frequency, 2 physical activity measurements and very brief advice (control), MVPA moderate-to-vigorous physical activity, AAS Active Australia Survey, PA physical activity, VM accelerometer vector magnitude

physical fitness and physical activity are closely linked and are predictors of all-cause mortality and cardiovascular events $[39,40]$.

There are important caveats that need to be considered when interpreting findings from the present study. Very brief physical activity interventions, or physical activity measurement alone, may require a longer intervention period to result in changes in physical activity behaviour. According to social cognitive theory, for an increase in physical activity to be adopted and maintained it must be sustained for at least 6-months [41]. Thus, the 18-week time period used in this study may not have been of sufficient length to allow for sustained changes in physical activity behaviour. In addition,
Waters et al. (2012) and Opendeacker et al. (2011) found that control group changes in physical activity were more likely when follow-up assessments were carried out over a longer period of time. Waters et al. (2012) found in their systematic review that follow-up assessments completed at 9 months as compared to 7 months were more likely to result in a clinically meaningful improvement in physical activity in control groups. Opendecker et al. (2011) found at 2-years, with a 12-month no intervention follow-up, there was no difference in aerobic fitness between 2 intervention groups (structured vs lifestyle) and a control group, concluding this was consistent with the control group improvement in physical activity and this was possibly due to a

Table 3 Measures of disease risk and fitness at baseline and 18-weeks

\begin{tabular}{|c|c|c|c|c|}
\hline \multirow[t]{2}{*}{ Characteristic } & \multicolumn{2}{|c|}{ Baseline mean (SD) } & \multicolumn{2}{|c|}{ 18-weeks mean (SD) } \\
\hline & HMF & LMF & HMF & LMF \\
\hline Waist circumference $(\mathrm{cm})$ & $84.7(12.6)$ & $85.2(15.1)$ & $82.3(11.5)^{\mathrm{A}}$ & $84(13)$ \\
\hline Waist-to-hip ratio & $0.79(0.08)$ & $0.81(0.09)$ & $0.79(0.08)$ & $0.80(0.8)$ \\
\hline Body mass index $\left(\mathrm{kg} / \mathrm{m}^{2}\right)$ & $28.1(5.4)$ & $26.4(4.1)$ & $28(5.2)$ & $26.5(4.2)$ \\
\hline $\mathrm{SBP}(\mathrm{mmHg})$ & $126(10)^{B}$ & $119(9)$ & $123(9)$ & $119(10)$ \\
\hline $\mathrm{DBP}(\mathrm{mmHg})$ & $82(10)$ & $77(9)$ & $78(7)^{\mathrm{A}}$ & $76(7)$ \\
\hline AQoL-6D utility score & $82.9(7.9)$ & $84.8(7.9)$ & $84.4(6.7)$ & $85.9(5.8)$ \\
\hline AQoL-6D Independent Living & $95(9.2)$ & $94.8(6.4)$ & $98.3(4.5)^{\mathrm{A}}$ & $99.3(2.5)$ \\
\hline AQoL-6D Relationships & $93(8.7)$ & $90.5(13.2)$ & $94.5(6.9)$ & $92.9(7.1)$ \\
\hline AQoL-6D Mental Health & $74(14)$ & $71.9(13.7)$ & $74.4(11.8)$ & $73.2(11.9)$ \\
\hline AQoL-6D Coping & $72(13)$ & $75(12.7)$ & $72.5(10.9)$ & $76.6(8)$ \\
\hline AQoL-6D Pain & $79.6(21.4)$ & 85.5 (19.6) & $79.5(26.5)$ & $89.7(18)$ \\
\hline AQoL-6D Senses & $81.9(10.4)$ & $86.5(10.8)$ & $84.2(9.2)$ & $83.3(8.7)$ \\
\hline STEP predicted $\mathrm{VO}_{2} \max (\mathrm{ml} / \mathrm{kg} / \mathrm{min})$ & $40.2(11.9)$ & $45.1(8.9)$ & $43.6(13.3)^{C}$ & $47.5(10.8)^{\complement}$ \\
\hline
\end{tabular}


measurement effect. Therefore, future physical activity measurement studies should consider measuring physical activity over a 6-month or greater period according to the social cognitive theory and improvements of physical activity in control groups.

Uncertainty remains about the most beneficial physical activity intervention components in adults, in terms of intervention length, intensity and mode, for example, individual versus group-based, face-to-face versus remotely delivered $[42,43]$. What is known is that most physical activity interventions work while they are being conducted, with positive results unlikely to be sustained over the longer term [44]. Professional background of those who deliver the physical activity intervention does not seem to influence the outcome but frequency of contacts might [42]. Considering the reach of health professionals around the world, further research is indicated to determine if an increased frequency of very brief physical activity interventions can increase adults' physical activity levels. If very brief physical activity interventions by physiotherapists are found to be successful, it may be a low cost efficient and effective method to increase a proportion of the populations' physical activity levels, leading to improved health throughout adulthood and into older age.

\section{Limitations and strengths}

There are many strengths of this pilot study such as the use of randomisation, concealed allocation, assessor blinding, objective measurement of physical activity, all participants being assessed over the same time period to eliminate a seasonal effect and use of intention-to-treat (primary) analyses, as well as a (secondary) per-protocol analysis for objectively measured physical activity. However, findings from this study should be interpreted with caution as the sample size was small, valid final accelerometer results were only provided by $75 \%(n=30 / 40)$ of the sample and the participants were predominantly educated women in a relationship, and dissimilar samples may not have responded in the same way. A medium effect size was found for the higher-measurement-frequency group which is useful for future sample size calculations. Although, it is unclear whether it was the additional physical activity measurements or the additional contacts with the physiotherapist that may have made a difference to physical activity levels. Furthermore, the intervention appeared to be feasible, requiring little time to implement (approximately $5 \mathrm{~min}$ for the aerobic fitness measure and physical activity advice), and limited space and equipment.

\section{Conclusion}

Frequency of physical activity measurement with very brief advice by physiotherapists may be enough to improve health outcomes in insufficiently active community-dwelling adults.
Further research is indicated with larger sample sizes and longer follow-up to determine whether increased frequency of very brief physical activity interventions by physiotherapists results in increased physical activity levels in community-dwelling adults.

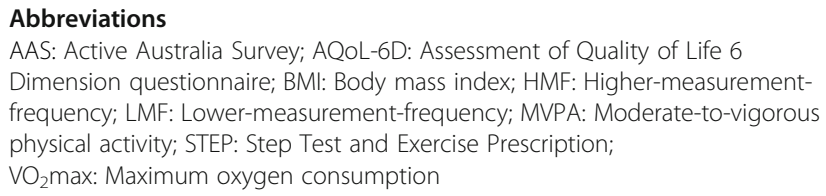

\section{Acknowledgements}

We are grateful to all the participants who took part in this study. We would also like to sincerely thank Nick Kershaw for his support as a research assistant on this project.

\section{Funding}

Funding was provided by the University of Canberra Health Research Institute. The funding body had no role in the design of the study;

collection, analysis, and interpretation of data; and in writing the manuscript.

\section{Availability of data and materials}

The datasets used and/or analysed during the current study are available from the corresponding author on reasonable request.

\section{Authors' contributions}

All authors contributed to the design of the study (NF, RD, SM). NF collected the data at baseline and 18-weeks. NF and SM analysed the data. All authors interpreted the data (NF, RD, SM). NF drafted the manuscript. All authors contributed to, read and approved the final manuscript (NF, RD, SM).

Ethics approval and consent to participate

Ethics approval was obtained from the University of Canberra Human Research Ethics Committee (HREC 16-30) in March 2016.

Study information including the project aim, data storage and details regarding participant involvement, confidentiality and anonymity were provided at the beginning of the study. All participants provided written consent after reading this information.

\section{Consent for publication}

Not applicable.

\section{Competing interests}

NF and RD declare that they have no competing interests. SM is an editorial board member of BMC Sports Science Medicine and Rehabilitation.

\section{Publisher's Note}

Springer Nature remains neutral with regard to jurisdictional claims in published maps and institutional affiliations.

\section{Author details}

${ }^{1}$ Physiotherapy, Faculty of Health, University of Canberra, Bruce 2617, Australia. ${ }^{2}$ Centre for Research \& Action in Public Health, University of Canberra, Bruce 2617, Australia. ${ }^{3}$ School of Public Health \& Social Work and Institute of Health and Biomedical Innovation, Queensland University of Technology, Victoria Park Road, Kelvin Grove 4059, Australia. ${ }^{4}$ Centre for Functioning and Health Research, Metro South Health, Corner of Ipswich Road and Cornwall Street, Buranda 4103, Australia.

Received: 26 June 2018 Accepted: 1 May 2019

Published online: 22 May 2019

References

1. Australian Bureau of Statistics. National Health Survey: first results 2014-15. Canberra: Australian Bureau of Statistics; 2015. http://www.abs.gov.au/ ausstats/abs@.nsf/Lookup/by\%20Subject/4364.0.55.001 2014-15 Main\%20Features Exercise 29 
2. Sallis JF, Bull F, Guthold R, Heath GW, Inoue S, Kelly P, et al. Progress in physical activity over the Olympic quadrennium. Lancet. 2016;388(10051): 1325-36. https://doi.org/10.1016/S0140-6736(16)30581-5.

3. Lear SA, Hu W, Rangarajan S, Gasevic D, Leong D, labal R, et al. The effect of physical activity on mortality and cardiovascular disease in 130000 people from 17 high-income, middle-income, and low-income countries: the PURE study. Lancet. 2017;390(10113):2643-54. https://doi.org/10.1016/S01406736(17)31634-3.

4. Stamatakis E, Murray A. Launch of new series: bright spots, physical activity investments that work. Br J Sports Med 2017; 51;19:1388-1388; doi:https:// doi.org/10.1136/bjsports-2017-098096.

5. United Nations. World population prospects: the 2017 revision, key findings and advance tables. New York: Department of Economic and Social Affairs Population Division; 2017.

6. Lee IM, Shiroma EJ, Lobelo F, Puska P, Blair SN, Katzmarzyk PT, et al. Effect of physical inactivity on major non-communicable diseases worldwide: an analysis of burden of disease and life expectancy. Lancet. 2012; 380 North American Edition;9838:219-29. https://doi.org/10.1016/S0140-6736(12)61031-9.

7. National Heart Foundation of Australia. Blueprint for an active Australia. 2nd ed. Melbourne: National Heart Foundation of Australia; 2014. https://www. heartfoundation.org.au/images/uploads/publications/Blueprint/Blueprint Health_care.pdf.

8. World Health Organisation: Draft WHO global action plan on physical activity 2018-2030. 2017; http://www.who.int/ncds/governance/gappa_ version_4August2017.pdf?ua=1.

9. World Health Organisation. Physical activity strategy for the WHO European region 2016-2025. Copenhagen: WHO Regional Office for Europe; 2016. http://www.euro.who.int/_data/assets/pdf_file/0014/311360/Physicalactivity-strategy-2016-2025.pdf?ua=1

10. Orrow G, Kinmonth A-L, Sanderson S, Sutton S. Effectiveness of physical activity promotion based in primary care: systematic review and metaanalysis of randomised controlled trials. BMJ 2012; 344:e1389-e1389; doi: https://doi.org/10.1136/bmj.e1389.

11. Mueller K. Communication from the inside out : strategies for the engaged professional. Philadelphia: F. A. Davis Company; 2010.

12. Kunstler BE, Cook JL, Freene $N$, Finch CF, Kemp JL, O'Halloran PD, et al. Physiotherapist-led physical activity interventions are efficacious at increasing physical activity levels: a systematic review and meta-analysis. Clin J Sport Med. 2017. https://doi.org/10.1097/JSM.0000000000000447.

13. Freene N, Cools S, Bissett B. Are we missing opportunities? Physiotherapy and physical activity promotion: a cross-sectional survey. BMC Sports Sci Med Reh. 2017;9:1-8.

14. Holm I, Tveter AT, Moseng T, Dagfinrud H. Does outpatient physical therapy with the aim of improving health-related physical fitness influence the level of physical activity in patients with long-term musculoskeletal conditions? Physiotherapy. 2015;101(3):273-8. https://doi.org/10.1016/j.physio.2014.11.005.

15. Lowe A, Littlewood C, McLean S, Kilner K. Physiotherapy and physical activity: a cross-sectional survey exploring physical activity promotion, knowledge of physical activity guidelines and the physical activity habits of UK physiotherapists. BMJ Open Sport and Exercise Medicine. 2017;3:1. https://doi.org/10.1136/bmjsem-2017-000290.

16. Abaraogu UO, Edeonuh JC, Frantz J. Promoting physical activity and exercise in daily practice: current practices, barriers, and training needs of physiotherapists in eastern Nigeria. Physiother Can. 2016;68(1):37-45. https://doi.org/10.3138/ptc.2014-74.

17. Waters L, Reeves M, Fjeldsoe B, Eakin E. Control group improvements in physical activity intervention trials and possible explanatory factors: a systematic review. J Phys Act Health. 2012;9(6):884-95.

18. Opdenacker J, Delecluse C, Boen F. A 2-year follow-up of a lifestyle physical activity versus a structured exercise intervention in older adults. J Am Geriatr Soc. 2011;59(9):1602-11. https://doi.org/10.1111/j.1532-5415.2011. 03551.x.

19. Eakin E, Reeves M, Lawler S, Graves N, Oldenburg B, Del Mar C, et al. Telephone counseling for physical activity and diet in primary care patients. Am J Prev Med. 2009;36(2):142-9.

20. van Sluijs EM, van Poppel MN, Twisk JW, van Mechelen W. Physical activity measurements affected participants' behavior in a randomized controlled trial. J Clin Epidemiol. 59(4):404-11.

21. French DR, Sutton S. Reactivity of measurement in health psychology: how much of a problem is it? What can be done about it? Br J Health Psychol. 2010;15(3):453-68. https://doi.org/10.1348/135910710X492341.
22. Lamming L, Pears S, Mason D, Morton K, Bijker M, Sutton S, et al. What do we know about brief interventions for physical activity that could be delivered in primary care consultations? A systematic review of reviews. Prev Med. 2017;99:152-63. https://doi.org/10.1016/j.ypmed.2017.02.017.

23. Sports Medicine Australia: Pre-Exercise Screening System. 2005. https://sma. org.au/sma-site-content/uploads/2017/08/new_pre_screening.pdf.

24. Knight E, Stuckey MI, Petrella RJ. Validation of the step test and exercise prescription tool for adults. Can J Diabetes. 2014;38(3):164-71 168p. https:// doi.org/10.1016/j.jcjd.2014.03.007.

25. Australian Government: Make your move - sit less - be active for life! Edited by Department of Health; 2014; http://www.health.gov.au/internet/ main/publishing.nsf/Content/F01F92328EDADA5BCA257BF0001E720D/\$File/ brochure\%20PA\%20Guidelines_A5_18-64yrs.pdf.

26. Australian Government: Healthy eating for adults. Edited by National Health and Medical Research Council Department of Health and Ageing; 2013. https://www.eatforhealth.gov.au/sites/default/files/content/ The\%20Guidelines/n55g adult_brochure.pdf.

27. Australia's Physical Activity and Sedentary Behaviour Guidelines [http:// www.health.gov.au/internet/main/publishing.nsf/content/health-pubhlthstrateg-phys-act-guidelines].

28. National Institute for Health and Care Excellence: Physical activity: brief advice for adults in primary care. Edited by National Institute for Health and Care Excellence; 2013; https://www.nice.org.uk/guidance/ph44.

29. Freedson PS, Melanson E, Sirard J. Calibration of the computer science and applications, Inc. accelerometer. Med Sci Sports Exerc. 1998;30(5):777-81.

30. Australian Institute of Health and Welfare. The active Australia survey: a guide and manual for implementation, analysis and reporting. Canberra: Australian Institute of Health and Welfare; 2003. http://www.aihw.gov.au/ publication-detail/?id=6442467449

31. Brown WJ, Trost SG, Bauman A, Mummery K, Owen N. Test-retest reliability of four physical activity measures used in population surveys. J Sci Med Sport. 2004;7(2):205-15.

32. Brown WJ, Burton NW, Marshall AL, Miller YD. Reliability and validity of a modified self-administered version of the active Australia physical activity survey in a sample of mid-age women. Aust N Z J Public Health. 2008;32(6): $535-41$

33. Stuckey MI, Knight E, Petrella RJ. The step test and exercise prescription tool in primary care: a critical review. Crit Rev Phy Rehabil Med. 2012;24(1-2): 109-23. https://doi.org/10.1615/CritRevPhysRehabilMed.2013006823.

34. AQoL Assessment of quality of life [http://www.aqol.com.au/index.php/ scoring-algorithms/112-6d/231-spss-6d-psychometric]. Accessed 1 June 2018.

35. Hawthorne G, Osborne R. Population norms and meaningful differences for the assessment of quality of life (AQoL) measure. Aust N Z J Public Health. 2005:29(2):136-42.

36. Allen J, Inder KJ, Lewin TJ, Attia JR, Kelly BJ. Construct validity of the assessment of quality of life - 6D (AQoL-6D) in community samples. Health Qual of Life Outcomes. 2013;11(1):1-15. https://doi.org/10.1186/1477-7525$11-61$.

37. Arain M, Campbell MJ, Cooper CL, Lancaster GA. What is a pilot or feasibility study? A review ofcurrent practice and editorial policy. BMC Med Res Methodol. 2010;10:67-73.

38. Freene N, Cools S, Hills D, Bissett B, Pumpa K, Cooper G. A wake-up call for physical activity promotion in Australia: results from a survey of Australian nursing and allied health professionals. Aust Health Rev. 2017. https://doi. org/10.1071/AH16240

39. Kodama S, Saito K, Tanaka S, Maki M, Yachi Y, Asumi M, et al. Cardiorespiratory fitness as a quantitative predictor of all-cause mortality and cardiovascular events in healthy men and women: a meta-analysis. JAMA. 2009;301(19):2024-35. https://doi.org/10.1001/jama.2009.681.

40. Warburton DER, Nicol CW, Bredin SSD. Health benefits of physical activity: the evidence. CMAJ. 2006;174(6):801-9. https://doi.org/10.1503/cmaj.051351.

41. Marcus BH, Forsyth LH, Stone EJ, Dubbert PM, McKenzie TL, Dunn AL, et al. Physical activity behavior change: issues in adoption and maintenance. Health Psychol. 2000;19(1):32-41.

42. Zubala A, Macgillivray S, Frost $H$, Kroll T, Skelton DA, Gavine A, et al. Promotion of physical activity interventions for community dwelling older adults: a systematic review of reviews. PLoS One. 2017;12(7):1-36. https:// doi.org/10.1371/journal.pone.0180902.

43. Greaves CJ, Sheppard KE, Abraham C, Hardeman W, Roden M, Evans PH, et al. Systematic review of reviews of intervention components associated 
with increased effectiveness in dietary and physical activity interventions. BMC Public Health. 2011;11(1):119. https://doi.org/10.1186/1471-2458-11-119.

44. Murray JM, Brennan SF, French DP, Patterson CC, Kee F, Hunter RF.

Effectiveness of physical activity interventions in achieving behaviour

change maintenance in young and middle aged adults: a systematic review and meta-analysis. Soc Sci Med. 2017;192:125-33. https://doi.org/10.1016/j. socscimed.2017.09.021

Ready to submit your research? Choose BMC and benefit from:

- fast, convenient online submission

- thorough peer review by experienced researchers in your field

- rapid publication on acceptance

- support for research data, including large and complex data types

- gold Open Access which fosters wider collaboration and increased citations

- maximum visibility for your research: over $100 \mathrm{M}$ website views per year

At $B M C$, research is always in progress.

Learn more biomedcentral.com/submissions 